worse on $62 \%$ of the variables and better on $0 \%$, while LMG was worse on $2.5 \%$ and better on $2.5 \%$ of variables. (Meador KJ, Loring DW, Ray PG et al. Differential cognitive and behavioral effects of carbamazepine and lamatrogine. Neurology May (1 of 2) 2001;56:1177-1182). (Reprints: Dr KJ Meador, Department of Neurology, Medical College of Georgia, 1120 15th St (BA3410), Augusta, GA).

COMMENT. Lamotrigine in healthy adult volunteers has fewer adverse cognitive and behavioral effects than carbamazepine at midrange standard anticonvulsant doses. Cognitive and behavioral side effects of AEDs are significant factors in decision to treat and duration of therapy of seizure disorders, especially in children. Equally important is a neuropsychological impairment that may be associated with the epilepsy syndrome, independent of any effects of AEDs.

\title{
NEUROCOGNITIVE PROFILE OF ABSENCE EPILEPSY SYNDROME
}

Cognitive and language function was determined in 16 children (mean age, 9.2 years; range 6-16) with absence epilepsy compared to 16 controls at the University of Catania, Italy. Children with absence epilepsy had subtle but significant deficits in global cognitive functioning (median full-scale IQ $90.8 \mathrm{cf}$ 103.2 in controls), and in visuospatial skills, nonverbal memory and delayed recall, while verbal memory and language function was preserved. Patients with early-onset seizures (< age 4 years) had more severe cognitive deficits than those whose epilepsy developed after age 4 years. (Pavone P. Bianchini R, Trifiletti RR et al. Neuropsychological assessment in children with absence epilepsy. Neurology April (2 of 2) 2001;56:1047-1051). (Reprints: Dr Piero Pavone, Divisione di Neuologia Pediatrica, Clinica Pediatrica, Universita di Catania, Viale Andrea 6, 95125 Catania, Italy).

COMMENT. Possible factors responsible for the impaired cognitive functioning in absence epilepsy syndrome include the effects of the seizures, the frequency and duration of the seizures, the underlying cause of the epilepsy, and the cognitive effects of antiepileptic drugs. The majority of patients in this study were treated with valproate monotherapy, and the possible adverse effects of the anticonvulsant cannot be discounted. (See Ped Neur Briefs Dec 2000;14:92, for report of study showing adverse effects of valproate on learning, memory, and behavior (Ronen et al. 2000)). The long-term follow-up of patients, comparing those whose seizures remit early and those requiring persistent therapy, would assist in differentiating the cause or causes of the cognitive dysfunction.

\section{SUPPRESSION OF INTERICTAL EPILEPTIC ACTIVITY BY AEDS}

Rates of full suppression of interictal epileptiform activity were compared for phenobarbital (PHB), carbamazepine (CBZ), and valproate (VPA), in a study at Tufts University School of Medicine, Boston, MA. Comparing 213 pairs of EEGs, overall suppression rates of epileptiform activity in the second EEG were 12/55 (22\%) for PHB, 27/81 (33\%) for CBZ, and 35/77 (46\%) for VPA (P=.005 for VPA vs PHB). Comparing EEG pairs with only generalized or focal discharges, VPA and CBZ were superior to PHB in suppressing generalized interictal epileptiform activity (47\%, 38\%, and $17 \%$, respectively) and focal discharges $(42 \%, 32 \%$, and $23 \%$, respectively). Comparing EEG pairs whose inter-EEG interval was less than 1 year, VPA and CBZ were equally effective and superior to PHB in suppressing generalized discharges $(46 \%, 50 \%$, and $14 \%$, respectively), whereas VPA was superior to both CBZ and PHB in suppressing focal discharges $(40 \%, 22 \%$, and $19 \%$, respectively). (Libenson $\mathrm{MH}$, Caravale $\mathrm{B}$. Do antiepileptic drugs differ in suppressing interictal epileptiform activity in children? Pediatr Neurol March 
2001;24:214-218). (Respond: Dr Libensen, Division of Pediatric Neurology, Floating Hospital for Children at New England Medical Center \#330, 750 Washington Street, Boston, MA 02111).

COMMENT. Valproate, and to a lesser degree carbamazepine, are superior to phenobarbital in completely suppressing interictal EEG seizure activity in children with epilepsy. Whereas VPA and CBZ are equally effective in controlling generalized discharges, VPA is superior to both CBZ and PHB in suppressing focal EEG discharges, especially when the interval between EEGs is less than 1 year. VPA is also superior to ethosuximide in suppressing generalized epileptiform discharges in children with absence seizures (personal observation).

\section{GABAPENTIN ADD-ON THERAPY FOR PARTIAL SEIZURES}

The efficacy and safety of gabapentin (GBP) as add-on therapy in 237 children, aged 3 to 12 years, with refractory partial seizures were studied at the Alder Hey Children's Hospital, Liverpool, UK. In a multicenter, open-label trial over a 6 month period, efficacy of GBP was evaluated by comparing the frequency of partial seizures during a 6-12 week base-line phase of a previous double-blind study to the frequency during the follow-up study. The median percent decrease in seizure frequency was $34 \%$ and the overall response rate $(>50 \%$ reduction in seizures) was $34 \%$. Six percent of patients withdrew because of adverse events, and $20 \%$ because of lack of efficacy. Concurrent AEDs were maintained in 78\%; doses were decreased in $11 \%$ and increased in $11 \%$. (Appleton R, Fichtner K, LaMoreaux L et al. Gabapentin as add-on therapy in children with refractory partial seizures: a 24-week, multicentre, open-label study. Dev Med Child Neurol April 2001;43:269-273). (Respond: Dr Richard Appleton, Alder Hey Children's Hospital, Liverpool L12 2AP, UK).

COMMENT. Gabapentin is well tolerated and effective as add-on treatment for refractory partial seizures in children.

\section{LANGUAGE DISORDERS}

\section{LANDAU-KLEFFNER SYNDROME: COURSE AND OUTCOME}

The presentation, course and outcome of Landau-Kleffner syndrome (LKS) were studied in 18 children (11 girls, 7 boys) followed for a mean of 67 months at Guy's Hospital, London, UK. All showed receptive language regression and electrical status epilepticus in sleep (ESES). Mean age at onset was 4 years 9 months (range 25-84 months). The length of ESES (mean 44 months) was correlated significantly with length of period between onset of illness and onset of recovery, with length of daytime EEG abnormalities, seizure frequency, and period of seizures (mean 36 months). The correlation between language outcome and seizure activity was weaker than that with ESES. Receptive language outcomes for those whose ESES lasted $<3$ years was significantly better than those with ESES for $>3$ years. Behavior problems (ADHD and ODD) were common (50\%), especially in patients with frontal lobe EEG abnormalities. Behavior abnormality in the acute phase was not correlated with seizure frequency, length of ESES, length of daytime EEG abnormality, nor with subsequent language outcome; there was a mild association with lower IQ. All children had impaired short-term memory at follow-up. Recovery began at a mean age of 106 months, and the mean duration of acute involvement was 50 months. Receptive and expressive language outcomes were strongly correlated, but language and IQ measures showed weaker associations. Language outcome was normal in three. In children with ESES 